\title{
Kinetic instability of the dust acoustic mode in inhomogeneous, partially magnetized plasma with both positively and negatively charged grains
}

\author{
J. Vranjes* and S. Poedts ${ }^{\dagger}$ \\ Centre for Plasma Astrophysics, Celestijnenlaan 200B, 3001 Leuven, Belgium and Leuven Mathematical Modeling \\ and Computational Science Research Centre (LMCC), Celestijnenlaan 200B, 3001 Leuven, Belgium \\ (Received 17 December 2009; revised manuscript received 4 August 2010; published 31 August 2010)
}

\begin{abstract}
A purely kinetic instability of the dust acoustic mode in inhomogeneous plasmas is discussed. In the presence of a magnetic field, electrons and ions may be magnetized while at the same time dust grains may remain unmagnetized. Although the dynamics of the light species is strongly affected by the magnetic field, the dust acoustic mode may still propagate in practically any direction. The inhomogeneity implies a source of free energy for an instability that develops through the diamagnetic drift effects of the magnetized species. It is shown that this may be a powerful mechanism for the excitation of dust acoustic waves. The analysis presented in the work is also directly applicable to plasmas containing both positive and negative ions and electrons, provided that at least one of the two ion species is unmagnetized.
\end{abstract}

DOI: 10.1103/PhysRevE.82.026411

\section{INTRODUCTION}

Inhomogeneous plasmas imply the presence of a source of free energy that can make some plasma modes grow. Within the kinetic theory a suitable distribution function for general plasma species $j$ and for an inhomogeneous plasma with a density gradient in the negative $x$ direction can be taken in the form [1]

$$
\begin{aligned}
f_{j 0}(x, \vec{v})= & N_{j}\left(\frac{m_{j}}{2 \pi \kappa T_{j}}\right)^{3 / 2} \exp \left\{-\left[v_{x}^{2}+\left(v_{y}+\frac{\kappa T_{j}}{m_{j} \Omega_{j} L_{j}}\right)^{2}\right.\right. \\
& \left.\left.+v_{z}^{2}\right] m_{j} /\left(2 \kappa T_{j}\right)-x / L_{j}\right\} .
\end{aligned}
$$

Here, the characteristic equilibrium density length is $L_{j}$ $=\left(n_{j 0}^{\prime} / n_{j 0}\right)^{-1}, n_{j 0}^{\prime}=d n_{j 0} / d x$, and $N_{j}=$ const. An integration over velocities yields the macroscopic equilibrium density profile $n_{j 0}(x)=\int f_{j 0} d^{3} \vec{v}=N_{j} \exp \left(-x / L_{j}\right)$. Similarly, one can also perform the integration of the type $J \equiv \int \vec{v} f_{j 0}(x, \vec{v}) d^{3} \vec{v}$. After a few steps this yields the equilibrium diamagnetic velocity corresponding to the expression standardly obtained from the fluid description $\vec{v}_{* j}=J /\left[n_{j 0}(x)\right]=-\kappa T_{j} /\left(m_{j} \Omega_{j} L_{j}\right) \vec{e}_{y}$ $\equiv \vec{e}_{y} \kappa T_{j} n_{j 0}^{\prime} /\left(q_{j} B_{0} n_{j 0}\right)$. It is seen that because $v_{* j} \sim T_{j} / q_{j}$, in the case of cool and/or highly charged species $j$, their diamagnetic effects may become negligible. This is sometimes a situation with ions in an electron-ion plasma where we have $\left|v_{* i} / v_{* e}\right|=\left(T_{i} / T_{e}\right)\left(e / q_{i}\right)$.

When both electrons and ions are magnetized, the corresponding growing low-frequency mode is the drift mode. However, in some situations, in the perturbed state the heavier species (ions) may, in fact, behave as unmagnetized regardless of the presence of a magnetic field. This may happen in case of high-frequency waves $\omega \gg \Omega_{i}=e B_{0} / m_{i}$ when the ions follow nearly straight lines, regardless of the mutual direction of the wave-number vector $\vec{k}$ from one side and the magnetic field vector from the other. In view of the mass difference, for electrons we may have at the same time $\omega$

\footnotetext{
*jovo.vranjes@wis.kuleuven.be

†stefaan.poedts@wis.kuleuven.be
}

PACS number(s): 52.27.Lw, 52.35.Fp, 52.35.Kt

$\ll \Omega_{e}=e B_{0} / m_{e}$. The ions can behave as unmagnetized in the perturbed state also in case of collisions, provided that $\nu_{i}$ $>\Omega_{i}$, even if at the same time $\Omega_{i}>\omega$. Similarly, they can behave as unmagnetized also for short wavelengths, $\lambda<\rho_{i}$, $\rho_{i}=v_{T i} / \Omega_{i}$, and $v_{T i}^{2}=\kappa T_{i} / m_{i}$. Electrostatic modes propagating in a plasma with such properties will have the basic features of the ion acoustic (IA) mode in spite of the magnetized electrons. Within the two-fluid theory such a mode in an inhomogeneous plasma may become growing [2-4] provided the simultaneous presence of collisions and the mentioned equilibrium density gradient perpendicular to $\vec{B}_{0}$. On the other hand, within the kinetic theory the mode is also growing due to purely kinetic effects. The growth rate is similar to the standard drift wave instability [5], with the necessary condition that the wave frequency is below the electron diamagnetic drift frequency $\omega_{* e}=v_{* e} k_{\perp}$.

In a plasma containing also dust grains and in the presence of some small perturbations, the time and space scales at which the grain dynamics develops are orders of magnitude different as compared to the above mentioned drift and IA wave scales. Hence, in situations similar to those described above, both the ions and electrons may be well magnetized, while the dust grains may not feel the effects of the magnetic field. One may then expect that the grain dynamics remains within the dust acoustic (DA) wave description although the lighter species are magnetized and thus have very different dynamics in the directions parallel and perpendicular to the magnetic field vector. If the plasma is inhomogeneous, the dynamics of the electrons and ions will be described by a set of equations that follow from the standard (kinetic or fluid) drift wave theory [6], with possibly the only difference regarding the finite ion inertia effects that at the DA wave scale can simply be ignored. The resulting dispersion equation may then yield a growing DA wave, with the instability driven by the density gradient of the magnetized light particles.

This type of the dust acoustic gradient-driven instability will be studied in the present work. In particular we shall use a model in which the equilibrium dusty plasma is confined by the magnetic field, implying that all species are magnetized, while the perturbations will be studied in time and 
space domains within which only lighter species (electrons and ions) are magnetized. Models of that kind are available in the literature $[7,8]$. Two specific cases will be studied: (i) an electron-ion plasma with negatively charged grains and (ii) a plasma containing positively charged grains, electrons, and negative ions.

\section{MODEL AND RESULTS}

\section{A. Plasma with negatively charged grains}

We shall assume a plasma placed in an external magnetic field $\vec{B}_{0}=B_{0} \vec{e}_{z}$, with a density gradient of the species $j$ in the $x$ direction $(j=e, i, d)$. The charge number $Z_{d}$ of the dust grains is assumed to be constant (which is appropriate for frequencies far below the mean attachment frequencies for electrons and ions $[9,10]$ ), so that the equilibrium quasineutrality condition reads

$$
n_{i 0}(x)=n_{e 0}(x)+Z_{d} n_{d 0}(x) .
$$

Details of the physics of the grain-plasma interaction and the corresponding charging may be found in Refs. [11,12]. The varying grain charge is known to introduce an extra mode of oscillations [9]. In such a geometry the species will have the above described equilibrium diamagnetic drift velocities $\vec{v}_{* j}$ $=\vec{e}_{z} \times \vec{\nabla} p_{j} /\left(q_{j} n_{j} B_{0}\right)$.

The perturbed number density of all three species $j$ may be calculated from

$$
n_{j 1}=\int_{\infty}^{\infty} f_{j 1} d^{3} \vec{v}
$$

where $f_{j 1}$ is the corresponding distribution function. In the perturbed state, we shall continue to treat the light species as magnetized. However, the dust grains are taken as unmagnetized, implying wavelengths that are above the ion and electron gyroradii yet below the grain gyroradius or/and the wave frequencies that are below the electron and ion gyrofrequency but above the grain gyrofrequency. Hence, the derivation will be different for magnetized and unmagnetized species. The former yields [6] for the perturbed number density,

$$
\begin{aligned}
n_{j 1}= & -\frac{q_{j} n_{j 0} \phi_{1}}{\kappa T_{j}}\left\{1+\left(\omega-\omega_{* j}\right) \sum_{m} \frac{\Lambda_{m}\left(\beta_{j}\right)}{\omega-m \Omega_{j}}\right. \\
& \left.\times\left[W\left(\frac{\omega-m \Omega_{j}}{\left|k_{z}\right| v_{T j}}\right)-1\right]\right\} .
\end{aligned}
$$

Here, $\quad \beta_{j}=k_{\perp}^{2} \rho_{j}^{2}, \quad \Lambda_{m}\left(\beta_{j}\right)=I_{m}\left(\beta_{j}\right) \exp \left(-\beta_{j}\right), \quad W(\chi)$ $=(2 \pi)^{1 / 2} \int_{-\infty}^{+\infty} \eta \exp \left(-\eta^{2} / 2\right) d \eta /(\eta-\chi)$, and $I_{m}$ is the modified Bessel function of the first kind and order $m$. We use the local approximation and the Fourier analysis with small perturbations of the form $\sim \hat{f}(x) \exp \left(-i \omega t+i k_{y} y+i k_{z} z\right)$, where $\hat{f}(x)$ is the $x$-dependent amplitude and $|d / d x| \ll\left|k_{y}\right|$. For frequencies much below $\Omega_{j}$ we keep only the term $m=0$ and, being interested in the DA spatial and time scales, it is good enough to use $\Lambda_{0}\left(\beta_{j}\right) \simeq 1$ for both electrons and ions. For similar reasons $(|\chi| \ll 1)$ we shall use the approximate expression

$$
W(\chi) \simeq 1-\chi^{2}+\cdots+i(\pi / 2)^{1 / 2} \chi \exp \left(-\chi^{2} / 2\right) .
$$

This yields for electrons and ions,

$$
\frac{n_{j 1}}{n_{j 0}}=-\frac{q_{j} \phi_{1}}{\kappa T_{j}}\left[1+i\left(\frac{\pi}{2}\right)^{1 / 2} \frac{\omega-\omega_{* j}}{\left|k_{z}\right| v_{T j}} \exp \left(\frac{\omega^{2}}{2 k_{z}^{2} v_{T j}^{2}}\right)\right] .
$$

Regarding the perturbed dust dynamics, we shall assume frequencies satisfying the conditions $\omega \gg \Omega_{d}$ and $\omega \gg \omega_{* d}$. The latter is particularly easily satisfied for grains with a high charge number. Further in the text, these assumptions will be supported by the parameters which we use. In such a frequency limit Eq. (2) yields [13,14]

$$
\frac{n_{d 1}}{n_{d 0}}=\frac{e Z_{d} \phi_{1}}{\kappa T_{d}}\left[1-\mathcal{Z}\left(\frac{\omega}{k v_{T d}}\right)\right] \text {. }
$$

Here, $\mathcal{Z}(\alpha)=\left[\alpha /(2 \pi)^{1 / 2}\right] \int_{-\infty}^{+\infty} d \xi \exp \left(-\xi^{2} / 2\right) /(\alpha-\xi)$ is the plasma dispersion function. Note that the argument $\alpha$ here comprises the total $k$, which describes the essential fact that the grain perturbations are independent on the direction of the magnetic field vector $\vec{B}_{0}=B_{0} \vec{e}_{z}$. After expanding $\mathcal{Z}(\alpha)$ $\simeq 1+1 / \alpha^{2}+\cdots-i(\pi / 2)^{1 / 2} \alpha \exp \left(-\alpha^{2} / 2\right)$, which is valid if $|\alpha| \gg 1$ and $|\operatorname{Re}(\alpha)| \gg|\operatorname{Im}(\alpha)|$, and using Eqs. (5) and (6), the quasineutrality condition, which in the present case reads $n_{i 1}=n_{e 1}+Z_{d} n_{d 1}$, yields the dispersion equation,

$$
\begin{aligned}
\Delta(\omega, \vec{k}) \equiv & \frac{k^{2} Z_{d}^{2} n_{d 0}}{m_{d} \omega^{2}}-\frac{n_{i 0}}{\kappa T_{i}}-\frac{n_{e 0}}{\kappa T_{e}}-i\left(\frac{\pi}{2}\right)^{1 / 2}\left[\frac{\omega}{k v_{T d}} \frac{Z_{d}^{2} n_{d 0}}{\kappa T_{d}}\right. \\
& \times \exp \left(-\frac{\omega^{2}}{2 k^{2} v_{T d}^{2}}\right)+\frac{\left(\omega-\omega_{* i}\right)}{k_{z} v_{T i}} \frac{n_{i 0}}{\kappa T_{i}} \exp \left(-\frac{\omega^{2}}{2 k_{z}^{2} v_{T i}^{2}}\right) \\
& \left.+\frac{\left(\omega-\omega_{* e}\right)}{k_{z} v_{T e}} \frac{n_{e 0}}{\kappa T_{e}} \exp \left(-\frac{\omega^{2}}{2 k_{z}^{2} v_{T e}^{2}}\right)\right]=0 .
\end{aligned}
$$

The real part of Eq. (7) yields the DA wave frequency $[15,16]$,

$$
\omega_{r}^{2}=k^{2} c_{d}^{2}, \quad c_{d}^{2}=\kappa T_{e} T_{i} n_{d 0} Z_{d}^{2} /\left[m_{d}\left(n_{i 0} T_{e}+n_{e 0} T_{i}\right)\right] .
$$

The growth rate (and damping) $\gamma$ is given approximately by $\gamma \simeq-\operatorname{Im}(\Delta) /[\partial \operatorname{Re}(\Delta) / \partial \omega]_{\omega=\omega_{r}}$, and this yields

$$
\begin{aligned}
\gamma= & -\left(\frac{\pi}{2}\right)^{1 / 2} \frac{m_{d} c_{d}^{3} k}{2 Z_{d}^{2} n_{d 0}}\left[\frac{\omega_{r} n_{d 0} Z_{d}^{2}}{k v_{T d} \kappa T_{d}} \exp \left(-\frac{\omega_{r}^{2}}{2 k^{2} v_{T d}^{2}}\right)\right. \\
& +\frac{\left(\omega_{r}-\omega_{* i}\right)}{k_{z} v_{T i}} \frac{n_{i 0}}{\kappa T_{i}} \exp \left(-\frac{\omega_{r}^{2}}{2 k_{z}^{2} v_{T i}^{2}}\right) \\
& \left.+\frac{\left(\omega_{r}-\omega_{* e}\right)}{k_{z} v_{T e}} \frac{n_{e 0}}{\kappa T_{e}} \exp \left(-\frac{\omega_{r}^{2}}{2 k_{z}^{2} v_{T e}^{2}}\right)\right] .
\end{aligned}
$$

The diamagnetic drift separates charges so that in the present case $\omega_{* i}$ has the sign opposite to $\omega_{* e}$. The shape of Eq. (9) reveals that, for a positive $\omega_{* e}$, the only term capable of changing the sign of $\gamma$ and yielding the growth is, in principle, the electron term, provided that $\omega_{r}<\omega_{* e}$. The dust term in Eq. (9) is typically always negligible in comparison to others. To check if the instability is really possible, we make the ratio of the ion and electron terms $\gamma_{i} / \gamma_{e}$ that are within the brackets in Eq. (9). As long as $\omega_{r}^{2} \ll k_{z}^{2} v_{T e}^{2}, k_{z}^{2} v_{T i}^{2}$ [see Eq. (4)], the exponential terms are practically equal to 
unity and play no role. Also, having $\omega_{r} \ll \omega_{* e}, \omega_{* i}$ it is seen that the instability may take place only provided that

$$
\left|\gamma_{i} / \gamma_{e}\right| \simeq\left|\frac{n_{i 0}^{\prime}}{n_{e 0}^{\prime}}\right|\left(\frac{T_{e} m_{i}}{T_{i} m_{e}}\right)^{1 / 2}=\left|1+\frac{Z_{d} n_{d 0}^{\prime}}{n_{e 0}^{\prime}}\right|\left(\frac{T_{e} m_{i}}{T_{i} m_{e}}\right)^{1 / 2}<1 .
$$

Hence, here the instability implies unrealistically high values of the ion temperature and the excitation of DA waves by electron terms in such dusty plasmas containing negatively charged grains is unlikely. An exception could be a specific case in which the electron and dust density gradients have opposite signs and, in addition, nearly balance each other so that the term $\left|1+Z_{d} n_{d 0}^{\prime} / n_{e 0}^{\prime}\right| \simeq\left|1-Z_{d} n_{d 0} L_{e} /\left(n_{e 0} L_{d}\right)\right|$ becomes very small, implying a nearly flat ion density profile, i.e., $L_{e} / L_{i} \ll 1$. The cases similar to that have been discussed in Refs. $[7,8]$. Note that in standard electron-ion plasma (e.g., in the case of the drift wave) exactly the same $\gamma_{e}$ term appears, resulting in the instability mentioned in the introductory lines, while at the same time it is usually $\omega_{r}^{2}>k_{z}^{2} v_{T i}^{2}$ or $\omega_{r}^{2}$ $\gg k_{z}^{2} v_{T i}^{2}$. The total ion contribution is thus considerably reduced and the electron terms are capable of making the mode growing. The reason for the absence of the electron-driven instability in the present dusty plasma case is clearly only due to the fact that the frequencies are low and the exponential term in the ion part plays no role, i.e., $\omega_{r}^{2} \ll k_{z}^{2} v_{T i}^{2}$.

\section{B. Positively charged grains and negative ions}

Under typical laboratory conditions in the past, grains are negatively charged by electron attachment, and this corresponds to the case discussed above. However, more recent experiments dealing with the photoelectric charging [17] and the presence of negative ions in a dusty plasma $[18,19]$ yield positively charged dust grains or both positive and negative at the same time. In space and astrophysical plasmas, grains can have both positive and negative charges, sometimes even at the same time or in the form of neighboring layers containing opposite charges on the grains [20,21]. This peculiar behavior of a dusty plasma is due to several competing charging mechanisms, such as the attachment of plasma particles due to inelastic collisions, secondary electron emission, and photoemission [22-24]. Dusty plasmas containing negative ions imply a reduced charging of the grains due to electron attachment and dust grains can become positively charged, especially in the case when positive ions are much lighter than negative ones [18]. On the other hand, we may simply have a plasma without dust but containing both positive and negative ions and electrons [15]. In such cases the diamagnetic velocities of electrons and any other negatively charged magnetized species have the same sign, and therefore their destabilizing effects will add up.

In view of that, performing a similar procedure as above, it can easily be shown that such plasmas are additionally destabilized, with the effects of negatively charged magnetized species (ions or grains) being similar to those of electrons. In the equilibrium we now have $Z_{d} n_{d 0}(x)=n_{i 0}(x)$ $+n_{e 0}(x)$. Here, the density gradients of electrons and negative ions are assumed to be of the same sign, and the quasineu-

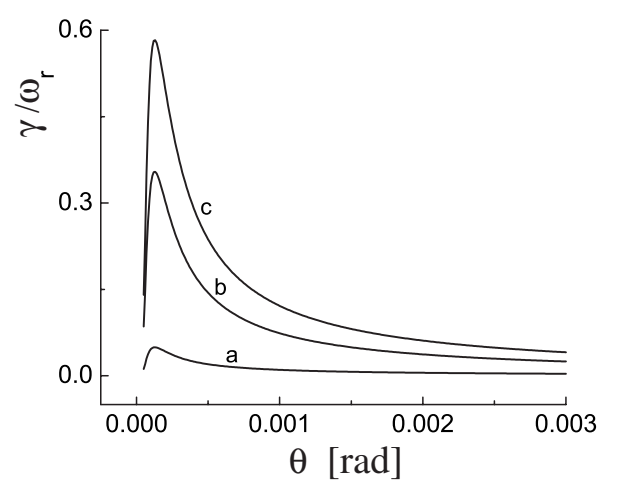

FIG. 1. The normalized growth rate $\gamma / \omega_{r}$ in terms of the angle between $\vec{k}$ and the $y$ axis for $T_{e}=T_{i}=0.1,0.5$, and $1 \mathrm{eV}$ (lines $a, b$, and $c$, respectively).

trality is maintained by the simultaneous variation of the density of positively charged grains. In the perturbed state the dispersion equation is obtained from

$$
Z_{d} n_{d 1}=n_{i 1}+n_{e 1} .
$$

Note that here the index $d$ may denote either positively charged grains or positive ions, while the index $i$ on the right-hand side in the present case describes any magnetized and negatively charged heavy species, ions, or grains.

The real part of the dispersion equation will again yield Eq. (8), while the growth rate will be similar as Eq. (9), only bearing in mind an essential difference as compared to the previously discussed case, i.e., that now both $\omega_{* j}$ in the critical terms $\omega-\omega_{* j}$ are positive. Hence, the mode is practically always unstable and the instability in the present case follows from the fact that both electrons and singly charged negative ions together change the sign of the growth rate $\gamma$.

Some additional features of this growing mode are presented in Figs. 1-3. In Fig. 1 we present the growth rate for positively charged grains and negative ions for an arbitrary set of plasma parameters and in terms of the angle of propagation $\theta=\arctan \left(k_{z} / k_{y}\right)$ between the wave vector $\vec{k}$ and the $y$ axis. The electron and negative-ion number densities are taken equal to $n_{i 0}=n_{e 0}=n_{0}=10^{18} \mathrm{~m}^{-3}$, and we take $Z_{d}=10^{3}$ yielding $n_{d 0}=2 \times 10^{15} \mathrm{~m}^{-3}$. We assume $m_{i}$ to be equal to

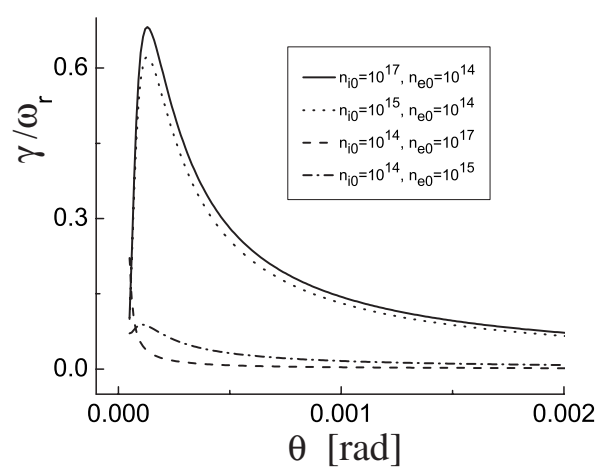

FIG. 2. The normalized growth rate $\gamma / \omega_{r}$ in terms of the angle between $\vec{k}$ and the $y$ axis for several values $n_{i 0}$ and $n_{e 0}$ and for $n_{d 0}=\left(n_{i 0}+n_{e 0}\right) / Z_{d}$. 


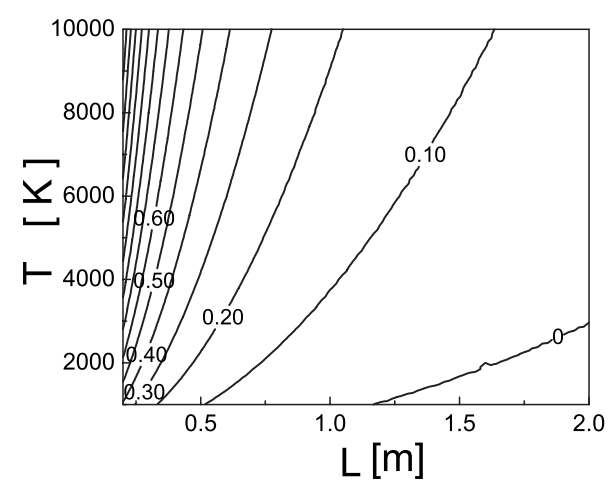

FIG. 3. The contour plot of the normalized growth rate $\gamma / \omega_{r}$ in terms of the inhomogeneity length $L=\left(d \ln n_{0} / d x\right)^{-1}$ and the plasma temperature.

proton mass, for grains we take $m_{d}=10^{-16} \mathrm{~kg}$, and we assume a plasma with $L \equiv L_{e}=L_{i}=\left(d \ln n_{0} / d x\right)^{-1}=1 \mathrm{~m}$. For the wave number we take $k=10^{3} \mathrm{~m}^{-1}$ so that $L / \lambda=160, \lambda$ $=2 \pi / k$, and $\rho_{d} / \lambda=3$, where $\rho_{d}=v_{T d} / \Omega_{d}$ and the magnetic field is taken as $B_{0}=0.2 \mathrm{~T}$.

The lines $a, b$, and $c$ in Fig. 1 correspond to three different values of the electron and ion temperatures, $T_{e}=T_{i}=T=0.1$, 0.5 , and $1 \mathrm{eV}$, respectively, where at the same time $T_{d}$ $=T / 50$. The growth rate is strongly dependent on both the angle of propagation and the temperature of the light plasma species that are responsible for the growth of the mode. Note that $\omega_{r}$ here takes values between 400 and $1260 \mathrm{~Hz}$ while $\Omega_{d}=0.3 \mathrm{~Hz}$, and $\omega_{* d}$ changes between 0.01 and $0.1 \mathrm{~Hz}$, so that indeed we have properly assumed limits for the local approximation, unmagnetized grains, and negligible dust diamagnetic drift effects.

We stress that taking any other value for the dust temperature, even going to the limit $T_{d} \sim T$, yields exactly the same shape for the growth rate. This is because for these parameters the dust contribution in Eq. (9) is completely negligible. Similarly, the growth rate does not change at all by varying $n_{0}$, and this holds as long as $n_{i 0}, n_{e 0}$ are kept equal. This may be expected from Eq. (9) because the density $n_{0}$ cancels out by the dust term $Z_{d} n_{d 0}$ that is in the denominator in front of the brackets. However, for any other values $n_{i 0} \neq n_{e 0}$ the growth rate changes. This is presented in Fig. 2 for several number densities $n_{i 0}, n_{e 0}$ (per cubic meter) and for the case $T=0.5 \mathrm{eV}, T_{d}=T / 50$. Here, the corresponding dust density is given by $Z_{d} n_{d 0}=n_{i 0}+n_{e 0}$.

The parameters above are used, in fact, in order to obtain a small enough growth rate so that the previously used expansions in Eq. (7) remain valid. Yet, for laboratory conditions the used value for $L$ is too large. Taking it smaller yields $\gamma>\omega_{r}$. Its variation strongly affects the growth rate, especially for a larger plasma temperature as seen from Fig. 3. Here, the contour plot of $\gamma\left(L_{n}, T\right)$ is presented for $T=T_{e}$ $=T_{i}$, and other parameters are as in Fig. 1. Obviously, the growth rate can easily be larger than the wave frequency, but this would imply that the conditions for the expansion used above are violated. In that case, in the above given quasineutrality condition (11) one should use general expressions (3) and (6) and solve the resulting dispersion equation numeri- cally. The result will almost always give strongly growing modes with $|\gamma| \gg\left|\omega_{r}\right|$.

\section{SUMMARY}

The described mechanism of excitation of dust acoustic waves in inhomogeneous plasmas with magnetized light species is very likely to occur in any inhomogeneous environment. In such situations, the DA wave will practically always be growing and the growth rate can easily be far above the wave frequency. The mode is most easily excited in a very narrow angle around a direction that is almost perpendicular to the magnetic field vector and the density gradient. In astrophysical clouds the proposed instability may become an initial triggering mechanism for the fragmentation, especially if self-gravity effects are also taken into account. The kinetic analysis presented here is, in fact, the most appropriate for such astrophysical plasmas with a small amount of collisions. Artificially produced grains under the laboratory conditions in the recent past are also positively charged [17-19], and the proposed instability discussed in Secs. II A and II B will undoubtedly work there too. However, plasmas in these recent experiments include not only positively charged grains, electrons, and negative ions, but, in addition to this, also a variable amount of positive ions [18] or negative grains [17]. The simplified model with three species only, discussed in Sec. II B, can easily be generalized to include the effects of these additional species.

The analysis performed in Sec. II A includes, as special case, some elements of the model presented in Refs. [7,8] where the ion density is taken constant, and the electron and grain densities are assumed to balance each other in order to preserve the quasineutrality, yet there are some remaining differences regarding the equilibrium. In our case, in both instabilities presented in Secs. II A and II B it is, in fact, the ion density gradient that crucially determines the mode behavior.

The model described in the text is, in fact, also directly applicable to plasmas free of dust but containing at the same time electrons together with both positively and negatively charged ions. One example of that kind [25] implies negative ions perfluoromethylcyclohexane $\left(\mathrm{C}_{7} \mathrm{~F}_{14}\right)$ and sulfur hexafluoride $\left(\mathrm{SF}_{6}\right)$, obtained by the electron attachment in a $\mathrm{Q}$ machine with an ionized potassium plasma.

The presented theory works in the standard limits of applicability of the kinetic theory. However, strongly collisional plasmas with a similar geometry and magnetization limits provide a counterpart instability driven by the simultaneous effects of the inhomogeneity and collisions. In ordinary electron-ion plasmas such an instability has been studied in the past [2-4].

\section{ACKNOWLEDGMENTS}

The results presented here are obtained in the framework of Projects No. G.0304.07 (FWO-Vlaanderen), No. C 90347 (Prodex), and No. GOA/2009-009 (K.U. Leuven). Financial support by the European Commission through the SOLAIRE Network (Grant No. MTRN-CT-2006-035484) is gratefully acknowledged. 
[1] P. M. Bellan, Fundamentals of Plasma Physics (Cambridge University Press, Cambridge, 2006), p. 260.

[2] N. A. Krall, in Advances in Plasma Physics, edited by A. Simon and W. B. Thompson (Interscience, New York, 1968), Vol. 1, p. 195.

[3] A. B. Mikhailovskii, Theory of Plasma Instabilities (Consultants Bureau, New York, 1974), Vol. 2, p. 192.

[4] J. Vranjes and S. Poedts, Phys. Plasmas 16, 022101 (2009).

[5] N. A. Krall and D. Book, Phys. Rev. Lett. 23, 574 (1969); Phys. Fluids 12, 347 (1969).

[6] J. Weiland, Collective Modes in Inhomogeneous Plasmas (Institute of Physics, Bristol, 2000).

[7] M. Rosenberg and P. K. Shukla, Plasma Phys. Controlled Fusion 46, 1807 (2004).

[8] M. Rosenberg and N. A. Krall, Phys. Plasmas 3, 644 (1996).

[9] J. Vranjes, B. P. Pandey, and S. Poedts, Phys. Rev. E 64, 066404 (2001).

[10] J. Vranjes, B. P. Pandey, and S. Poedts, Phys. Plasmas 9, 1464 (2002).

[11] B. T. Draine and B. Sutin, Astrophys. J. 320, 803 (1987).

[12] J. Vranjes, M. Y. Tanaka, B. P. Pandey, and M. Kono, Phys. Rev. E 66, 037401 (2002).
[13] J. Vranjes and S. Poedts, Phys. Plasmas 13, 052103 (2006).

[14] J. Vranjes and S. Poedts, Eur. Phys. J. D 40, 257 (2006).

[15] N. D'Angelo, S. V. Goeler, and T. Ohe, Phys. Fluids 9, 1605 (1966).

[16] N. N. Rao, P. K. Shukla, and M. Y. Yu, Planet. Space Sci. 38, 543 (1990).

[17] A. A. Sickafoose, J. E. Colwell, M. Horányi, and S. Robertson, Phys. Rev. Lett. 84, 6034 (2000).

[18] R. L. Merlino and Sun-Hyun Kim, Appl. Phys. Lett. 89, 091501 (2006).

[19] M. Rosenberg and R. L. Merlino, Planet. Space Sci. 55, 1464 (2007).

[20] O. Havnes, T. Aslaksen, and A. Brattli, Phys. Scr., T T89, 133 (2001).

[21] L. J. Gelinas, K. A. Lynch, M. C. Kelley, S. Collins, S. Baker, Q. Zhou, and J. S. Friedman, Geophys. Res. Lett. 25, 4047 (1998).

[22] B. T. Draine and E. E. Salpeter, Astrophys. J. 231, 77 (1979).

[23] N. D'Angelo, Planet. Space Sci. 49, 1251 (2001).

[24] A. A. Mamun, Phys. Rev. E 77, 026406 (2008).

[25] S.-H. Kim and R. L. Merlino, Phys. Rev. E 76, 035401 (2007). 